CALT-68-2718

IPMU-09-0004

\title{
Geometry As Seen By String Theory
}

\author{
Hirosi Ooguri \\ California Institute of Technology, Pasadena, CA 91125, USA \\ and \\ Institute for the Physics and Mathematics of the Universe, \\ University of Tokyo, Kashiwa, Chiba 277-8586, Japan
}

The Fourth Takagi Lectures of the Mathematical Society of Japan, delivered on 21 June 2008 at Department of Mathematics, Kyoto University 


\section{Introduction}

It is a great privilege to deliver this set of lectures in honor of Professor Teiji Takagi (1875 - 1960), the founding father of modern mathematical research in Japan. Professor Takagi was an alumnus of my high school, and our mathematics teacher liked to tell us about the local hero who realized Kronecker's Jugendtraum in the case of imaginary quadratic fields by establishing Class Field Theory [1]. As a high school student, I also enjoyed reading his popular book on the history of modern mathematics [2], where the moments of creation of new mathematics are vividly described. I was particularly fascinated by the story on elliptic functions of Gauss, Abel, and Jacobi, which turned out to be relevant in my study of conformal field theories 7 years later. We cannot overestimate the influence of his legendary textbook on Calculus [3] over generations of engineers and scientists as well as mathematicians in Japan for the last three quarters of a century since it was first published. On my bookshelves, it stands next to Feynman Lectures on Physics and Landau-Lifshitz Course of Theoretical Physics, and I still consult it from time to time.

The main subject of this set of lectures is the topological string theory. The topological string theory was introduced by E. Witten about 20 years ago, and it has been developed by collaborations of physicists and mathematicians. Its mathematical structure is very rich, and it has lead to discoveries of new connections between different areas of mathematics, ranging from algebraic geometry, symplectic geometry and topology, to combinatorics, probability and representation theory. The topological string theory also has many important applications to problems in physics. Though the theory was initially thought of as a simple toy model of string theory, it has turned out to be useful in computing a certain class of scattering amplitudes of physical string theory. In the past 10 years, the relation between topological string and physical string has been applied to variety of problems, and it has advanced our understanding of string compactifications, provided a powerful computational tool to study strongly coupled dynamics of gauge theories, has shed light on mysteries of quantum gravity such as quantum states of black holes, and pointed out a promising direction to prove the AdS/CFT correspondence. Moreover, the topological string theory has given us insights into how our concept of space and time should be modified in order to formulate fundamental laws of nature.

Although these lectures are meant to be for mathematicians, I felt it would be appropriate to spend the first couple of minutes in this course explaining physicists' motivation to study string theory. In the past few hundred years, physicists have searched for fundamental laws of nature by exploring phenomena at shorter and shorter distances. Although 
the idea that everything on the Earth is made of atoms goes back to Ancient Greek, the modern atomic theory began with the publication of "New System of Chemical Philosophy" by J. Dalton in 1808. In the middle of the 19th century, the size of atoms is correctly estimated to be about $10^{-10}$ meters. By the end of the 19 th century, due to the discovery of the electron and study of radioactivity, scientists began to think that atoms are not fundamental and that they have internal structure. In 1904, H. Nagaoka proposed the model in which there is a positively charged nucleus at the center with electrons orbiting around them. The existence of atomic nuclei was confirmed by the Geiger-Marsden experiment and the theory of E. Rutherford. The radius of the atomic nucleus is about $10^{-15}-10^{-14}$ meters. In the 1930th, thanks to the discovery of the neutron by J. Chadwick, the splitting of the atomic nucleus by J. Cockroft and E. Walton, and the meson theory of H. Yukawa, it became clear that the atomic nucleus is made of protons and neutrons bound together by the $\pi$ meson. The radius of the proton is about $10^{-15}$ meter. The progress of elementary particle physics in the past 50 years has culminated in the "Standard Model of Particle Physics," which describes all known particle physics phenomena down to $10^{-18}$ meters. The Large Hadron Collider, which just began its operation at CERN in Switzerland, will probe distance as short as $10^{-19}$ meters.

It is natural to ask whether this progression continues indefinitely. Surprisingly, there are reasons to think that the hierarchical structure of nature will terminate at the Planck length at $10^{-35}$ meters. Let us perform a thought-experiment to explain why this might be the case. Physicists build particle colliders to probe short distances. The more energy we use to collide particles, the shorter distances we can explore. This has been the case so far. One may then ask: can we build a collider with energy so high that it can probe distances shorter than the Planck length? The answer is no. When we collide particles with such high energy, a black hole will form and its event horizon will conceal the entire interaction area. Stated in another way, the measurement at this energy would perturb the geometry so much that the fabric of space and time would be torn apart. This would prevent physicists from ever seeing what is happening at distances shorter than the Planck length. This is a new kind of uncertainty principle. The Planck length is truly fundamental since it is the distance where the hierarchical structure of nature will terminate.

Space and time do not exist beyond the Planck scale, and they should emerge from a more fundamental structure. Superstring theory is a leading candidate for a mathematical framework to describe physical phenomena at this scale since it contains all the ingredients necessary to unify quantum mechanics and general relativity. 


\section{From Points to Strings}

The axiomatic method for geometry invented by Euclid of Alexandria is based on mathematical points with no size and structure. The Elements defines a point as "that which has no part." 2300 years after Euclid, string theory is offering the first real alternative to this approach by introducing finite-sized objects as basic building blocks.

Consider a Riemannian manifold $M$ and try to probe it using a point-like particle. A typical example of "observables" is Green's function $G(x, y)$ obeying

$$
\left(-\Delta_{x}+m^{2}\right) G(x, y)=\delta(x, y)
$$

where $x, y \in M, \Delta_{x}$ is the Laplace-Beltrami operator on $x, m$ is a constant, which physicists regard as a mass of the particle, and $\delta(x, y)$ is the delta-function for $x=y$. Green's function can be expressed as a path integral, i.e., a sum over all possible paths in $M$ from $x$ to $y$ with an appropriate weight.

In string theory, an analogue of Green's function has richer structure. An obvious analogue would be a sum over all possible spherical surface connecting $x$ to $y$ as in Figure 1(a). But there is no reason to stop at two points. We can choose $n$ point, $x_{1}, x_{2}, \cdots, x_{n}$, and sum over all spherical surfaces connecting them as in Figure 1(b). To define a similar object in a point particle theory, one would need to introduce "interactions." In string theory, interactions are already built in without additional assumptions. More generally, we can consider a sum over genus- $g$ surfaces connecting the $n$ points to define an amplitude $F_{g}\left(x_{1}, \cdots, x_{n}\right)$. We can go even further; since we are considering string theory, we should be able to consider $n$ configurations of strings in $M$ and a sum over genus- $g$ surfaces with $n$ boundaries connecting them as in Figure 1(c). This can be made a little more precise as follows. 


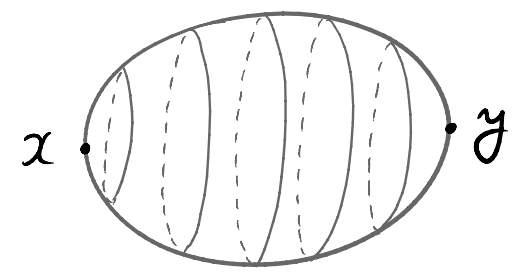

(a)

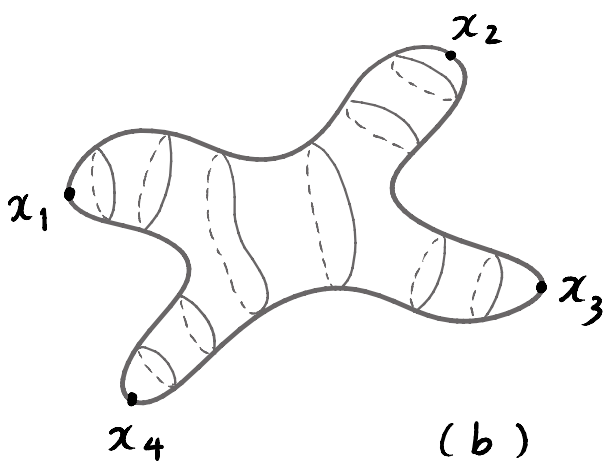

(b)

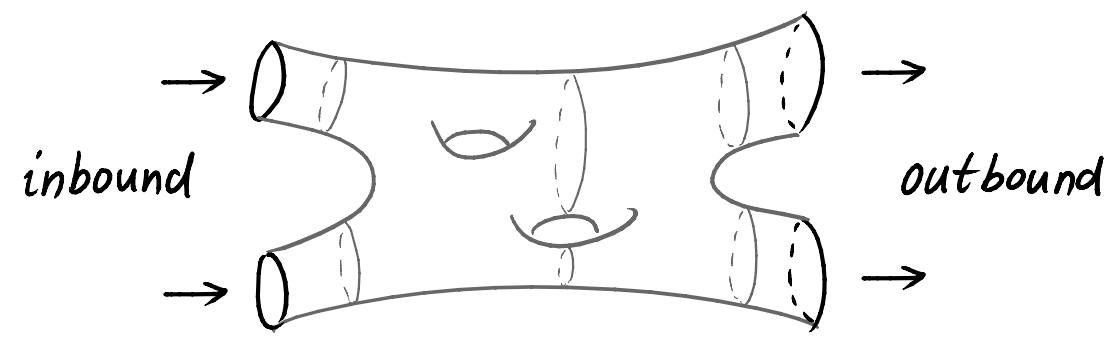

(c)

Figure 1 An analogue of Green's function in string theory is given by summing over spheres with two points fixed (a). This can be generalized to $n$-point functions (b) and to higher genus amplitudes with incoming and outgoing string states (c).

\subsection{String Amplitudes at Genus $g$}

Let us define a conformal field theory in two dimensions. We start with a Hilbert space $\mathcal{H}$, which realizes the Virasoro algebra

$$
\left[L_{n}, L_{m}\right]=(n-m) L_{n+m}+\frac{c}{12}\left(n^{3}-n\right) \delta_{n+m, 0}, \quad n, m=0, \pm 1, \pm 2, \cdots
$$

Physicists regard it as a "space of states" of the conformal field theory. Here $c$ is the central charge, which commutes with all other generators and takes a fixed value on $\mathcal{H}$. The Hilbert space $\mathcal{H}$ is decomposed into a sum of products of irreducible unitary representations $V_{h}$ of the Virasoro algebra with the highest weight $h$ as

$$
\mathcal{H}=\oplus_{h, \bar{h}} N_{h, \bar{h}} V_{h} \otimes V_{\bar{h}}
$$

where $N_{h, \bar{h}}$ are non-negative integers. It is convenient to distinguish the Virasoro algebra realized on the two factors of $V_{h} \oplus V_{\bar{h}}$, so physicists use $\left\{L_{n}\right\}$ for $V_{h}$ and $\left\{\bar{L}_{n}\right\}$ for $V_{\bar{h}}$ and call them the left-movers and the right-movers. 
According to [4,5], a conformal field theory is defined as a functor,

$$
\Sigma_{g}(n, m) \rightarrow \mathcal{A}_{g}(n, m) \in \operatorname{Hom}\left(\mathcal{H}^{\otimes n}, \mathcal{H}^{\otimes m}\right),
$$

where $\Sigma_{g}(n, m)$ is a Riemann surface of genus $g$ with $n$ parametrized boundaries with the inbound orientation and $m$ parametrized boundaries with the outbound orientation. It is supposed to satisfy the gluing axioms spelled out in [5].

To define string theory, we need a particular type of conformal field theory where one can define a nilpotent operator $Q: \mathcal{H} \rightarrow \mathcal{H}$ of degree 1 such that the Virasoro generators are $Q$-trivial,

$$
L_{n}=\left\{Q, b_{n}\right\}, \bar{L}_{n}=\left\{Q, \bar{b}_{n}\right\},
$$

for some operators $b_{n}, \bar{b}_{n}: \mathcal{H} \rightarrow \mathcal{H}$ of degree -1 . We need the central charge $c=0$ for this to be possible. In physics literature, $b_{n}$ 's are called anti-ghosts. Let $\mathcal{M}_{g}(n, m)$ be the moduli space of $\Sigma_{g}(n, m)$, and $\Omega^{*}\left(\mathcal{M}_{g}(n, m)\right)$ be the space of differential forms on it. When (2.5) holds for a conformal field field theory (2.4), one can define

$$
\omega_{g}(n, m) \in \Omega^{*}\left(\mathcal{M}_{g}(n, m)\right) \otimes \operatorname{Hom}\left(\mathcal{H}^{\otimes n}, \mathcal{H}^{\otimes m}\right),
$$

so that it is closed, $D \omega_{g}(n, m)=0$ with respect to $D=d+Q$, where $d$ is the de Rham operator on $\Omega^{*}\left(\mathcal{M}_{g}(n, m)\right)$. There is a set of gluing axioms for $\omega_{g}(n, m)$. For example, for the gluing map,

$$
\text { glue }: \mathcal{M}_{g_{1}}(n, k) \oplus \mathcal{M}_{g_{2}}(k, m) \rightarrow \mathcal{M}_{g_{1}+g_{2}}(n, m) \text {, }
$$

$\omega_{g}(n, m)$ responds as

$$
\begin{aligned}
& \text { glue }^{*}\left(\omega_{g_{1}+g_{2}}(n, m)\right)=\operatorname{Tr}_{\mathcal{H}}^{\otimes k}\left(\omega_{g_{1}}(n, k) \omega_{g_{2}}(k, m)\right) \\
& \in \Omega^{*}\left(\mathcal{M}_{g_{1}}(n, k) \oplus \mathcal{M}_{g_{2}}(k, m)\right) \otimes \operatorname{Hom}\left(\mathcal{H}^{\otimes n}, \mathcal{H}^{\otimes m}\right) .
\end{aligned}
$$

Physicists have developed a method to construct $\omega_{g}(n, m)$ for all known perturbative string theories, including bosonic string, superstring, heterotic string and topological string. The genus- $g$ string amplitude $F_{g}(n, m)$ is given by integrating the top component of $\omega_{g}(n, m)$ over $\mathcal{M}_{g}(n, m)$,

$$
F_{g}(n, m)=\int_{\mathcal{M}_{g}(n, m)} \omega_{g}(n, m) .
$$




\subsection{Open Strings}

Mathematics of open strings was pioneered by K. Fukaya and M. Kontsevich before physicists (except for Witten [6]) realized geometric significance of D branes. Although it is difficult to do justice to recent progress in this area's mathematics in this short course of lectures, let us briefly introduce D branes and open strings as we will need them later.

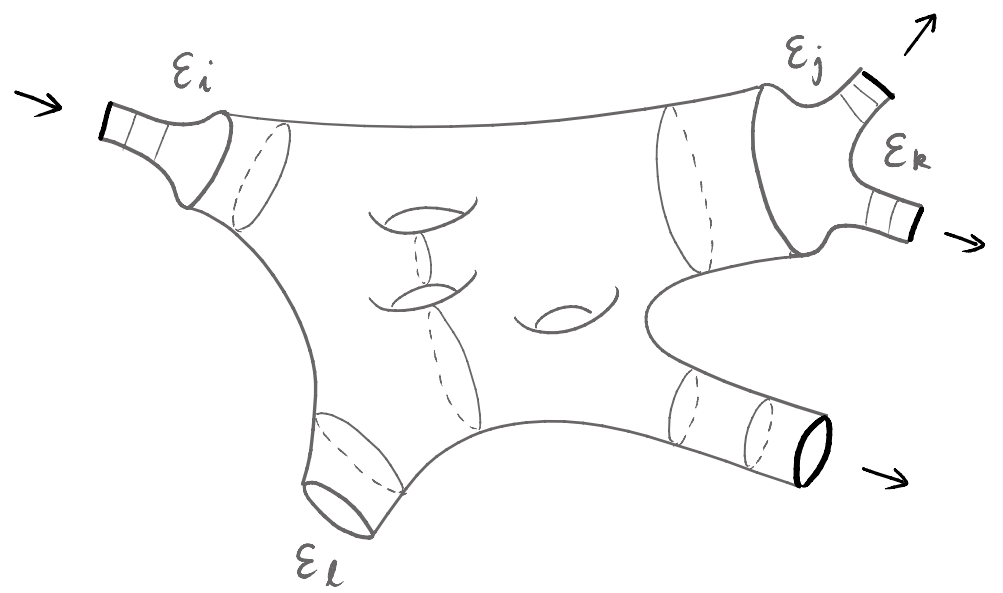

Figure 2 An open string amplitude of genus 3. It has no incoming closed string, 1 incoming open string, 1 outgoing closed string, and 2 outgoing open strings $\left(n=0, n_{\text {open }}=1, m=1, m_{\text {open }}=2\right)$. Note that each open string state is attached to a segment on a boundary. The remaining boundary components are assigned with boundary conditions, $\mathcal{E}_{i}, \mathcal{E}_{j}, \mathcal{E}_{k}, \mathcal{E}_{l}$.

An open string has two end-points, and we need to specify boundary conditions there. In physics literature, each boundary condition is called a D brane. Let us choose a set of $\mathrm{D}$ branes, $\left\{\mathcal{E}_{i}: i=1, \cdots, k\right\}$. Some of the $\mathrm{D}$ branes may coincide, i.e., some boundary conditions may be identical. For a pair of D branes, $\mathcal{E}_{i}$ and $\mathcal{E}_{j}$, we can define an open string Hilbert space $\mathcal{H}_{\text {open }}\left(\mathcal{E}_{i}, \mathcal{E}_{j}\right)$. Consider a Riemann surface $\Sigma_{g, b}\left(n, n_{\text {open }} ; m, m_{\text {open }}\right)$ with genus $g$ and $(b+n+m)$ boundaries with the following markings. See Figure 2 . As in the case of closed string, there are $n$ parametrized boundaries with the inbound orientation and $m$ parametrized boundaries with the outbound orientation. In addition, there are $\left(n_{\text {open }}+m_{\text {open }}\right)$ disjoint embeddings of the interval $[0,1]$ into the remaining $b$ boundaries, where $n_{\text {open }}$ of them are inbound and $m_{\text {open }}$ are outbound. We arrange so that boundaries make a right-angle turn at each end point of the images of $[0,1]$. This is needed for the gluing rules to work for open strings. The complement of these images in the $b$ boundaries has several disjoint components, and they are either circles or intervals. We 
assign a D brane boundary condition to each of the disjoint components. In this way, each image of $[0,1]$ is sandwiched by a pair of boundary components with prescribed boundary conditions, $\mathcal{E}_{i}$ and $\mathcal{E}_{j}$ for example. We can then associate the Hilbert space $\mathcal{H}_{\text {open }}\left(\mathcal{E}_{i}, \mathcal{E}_{j}\right)$ to the image on $[0,1]$ on the boundary. The open/closed conformal field theory is a functor,

$$
\begin{aligned}
\Sigma_{g, b}\left(n, n_{\text {open }} ; m, m_{\text {open }}\right) \rightarrow & \mathcal{A}_{g, b}\left(n, n_{\text {open }} ; m, m_{\text {open }}\right) \\
& \in \operatorname{Hom}\left(\mathcal{H}^{\otimes n} \otimes \mathcal{H}_{\text {open }}^{\otimes n_{\text {open }}}, \mathcal{H}^{\otimes m} \otimes \mathcal{H}_{\text {open }}^{\otimes m_{\text {open }}}\right) .
\end{aligned}
$$

If the Virasoro generator acting on $\mathcal{H}$ and $\mathcal{H}_{\text {open }}$ are $Q$-trivial, one can construct

$$
\begin{aligned}
\omega_{g, b}\left(n, n_{\text {open }} ; m, m_{\text {open }}\right) \in \Omega^{*}\left(\Sigma_{g, b}\left(n, n_{\text {open }} ; m, m_{\text {open }}\right)\right) & \\
& \otimes \operatorname{Hom}\left(\mathcal{H}^{\otimes n} \otimes \mathcal{H}_{\text {open }}^{\otimes n_{\text {open }}}, \mathcal{H}^{\otimes m} \otimes \mathcal{H}_{\text {open }}^{\otimes m_{\text {open }}}\right),
\end{aligned}
$$

which is closed with respect to $D=d+Q$, and it can be used to define a string amplitude $F_{g, b}\left(n, n_{\text {open }} ; m, m_{\text {open }}\right)$ by integrating its top component over the moduli space.

Open string theory and closed string theory (the one which contains closed strings only) seem very different. For one thing, open string theory can be formulated using string field theory [7]. Mathematically, it means that the moduli space for $\Sigma_{g, b \neq 0}$ has a nice triangulation that corresponds to a sum of Feynman diagrams. String field theories for open topological strings are particularly simple. In section 5, we will discuss the ChernSimons gauge theory in three dimensions [8] and random matrix models [9], as examples of string field theories. In contrast, a string field theory to compute closed string amplitudes $F_{g}$ (2.9) for all $g$ is not known, except for the topological B-model [10]. The topological $\mathrm{B}$-model is special since it is manifest that the string amplitudes $F_{g}$ receive contributions only from boundaries of the moduli space $\mathcal{M}_{g}$.

\section{Topological String Theory}

To define the topological string theory, we consider a conformal field theory with the $\mathcal{N}=2$ superconformal symmetry generated by $\left\{L_{n}, G_{n}^{+}, G_{n}^{-}, J_{n}\right\}$ and the central charge $\hat{c}$. These generators obey the commutation relations, 1

$$
\begin{aligned}
& {\left[L_{n}, L_{m}\right]=(n-m) L_{n+m},\left\{G_{n}^{+}, G_{m}^{-}\right\}=2 L_{n+m}} \\
& {\left[L_{n}, G_{m}^{+}\right]=-m G_{n+m}^{+},\left[L_{n}, G_{m}^{-}\right]=(n-m) G_{n+m}^{-},\left[J_{n}, G_{m}^{ \pm}\right]= \pm G_{n+m}^{ \pm}} \\
& {\left[J_{n}, J_{m}\right]=\hat{c} n \delta_{n+m},\left[L_{n}, J_{m}\right]=-m J_{n+m} .}
\end{aligned}
$$

1 A CFT connoisseur may notice that the commutation relations listed here are slightly difference from the standard ones. In fact, they are related to by rearranging the generators, the process called topological twist. 
We see that the Virasoro algebra with $c=0$ is a sub-algebra and that the Virasoro generators are $Q$-trivial, $L_{n}=\left\{Q, b_{n}\right\}$, where $Q=G_{0}^{+}+\bar{G}_{0}^{+}$and $b_{n}=\frac{1}{2} G_{n}^{-}$. Thus, we can use the formalism of section 2.2 to define the genus- $g$ string amplitudes $F_{g}(n, m)$.

\subsection{Two Models for One Calabi-Yau Manifold}

A prototypical example of conformal field theories with $\mathcal{N}=2$ superconformal symmetry is the supersymmetric sigma-model whose target space is a Calabi-Yau manifold $M$. For a given Calabi-Yau manifold, one can define two distinct sigma-models with different $Q$ operators, the A-model and the B-model [11].

In physics, the A-model is defined in terms of a path integral over (not necessarily holomorphic) maps

$$
X: \Sigma \rightarrow M
$$

together with Grassmannian fields on $\Sigma$,

$$
\begin{aligned}
& \theta(z, \bar{z}) \in T_{X(z, \bar{z})}^{1,0} M, \quad \eta(z, \bar{z}) \in \Omega^{1,0}(\Sigma) \otimes T_{X(z, \bar{z})}^{0,1} M, \\
& \bar{\theta}(z, \bar{z}) \in T_{X(z, \bar{z})}^{0,1} M, \quad \bar{\eta}(z, \bar{z}) \in \Omega^{0,1}(\Sigma) \otimes T_{X(z, \bar{z})}^{1,0} M,
\end{aligned}
$$

where $(z, \bar{z}) \in \Sigma, T_{x}^{1,0} M$ and $T_{x}^{0,1} M$ are the holomorphic and anti-holomorphic components of the tangent space at $x \in M$, and $\Omega^{1,0}(\Sigma)$ and $\Omega^{0,1}(\Sigma)$ are the spaces of $(1,0)$ and $(0,1)$ forms on $\Sigma$. In this model, the operator $Q$ is the Noether charge associated to the following transformation,

$$
\begin{aligned}
& \delta X^{i}=\epsilon \theta^{i}, \quad \delta \bar{X}^{\bar{i}}=\epsilon \bar{\theta}^{\bar{i}} ; \quad \delta \theta^{i}=\delta \bar{\theta}^{\bar{i}}=0 \\
& \delta \bar{\eta}^{i}=\epsilon \bar{\partial} X^{i}-\Gamma_{j k}^{i} \theta^{j} \bar{\eta}^{k}, \quad \delta \eta^{\bar{i}}=\epsilon \partial \bar{X}^{\bar{i}}-\Gamma_{\bar{j} \bar{k}}^{\bar{i}} \bar{\theta}^{\bar{j}} \eta^{\bar{k}}
\end{aligned}
$$

where I have used holomorphic coordinates $\left(x^{1}, \cdots, x^{\hat{c}}\right), \Gamma_{j k}^{i}$ is the Christoffel connection associated to the Ricci flat Kähler metric, and $\epsilon$ is a Grassmann number to parametrize the transformation. It then follows that $Q$ cohomology of $\mathcal{H}$ in this model is given by the Hodge-de Rham cohomology of $M$ as,

$$
\mathrm{H}_{Q}(\mathcal{H})=\oplus_{p, q=0}^{\hat{c}} \mathrm{H}^{p, q}(M)
$$

The degrees $(p, q)$ can be identified as eigenvalues of $\left(J_{0}, \bar{J}_{0}\right)$ in the left-moving and rightmoving $\mathcal{N}=2$ superconformal algebras.

Another consequence of the $Q$ symmetry is that a path integral for a $Q$ invariant amplitude localizes to a sum over fixed points of the symmetry.2 For the A-model, the

2 See section 5 of 11$]$ for discussion on the localization mechanism. 
fixed points of (3.4) consist of holomorphic maps from $\Sigma$ to $M$ together with $\theta=\bar{\theta}=0$, so that $\delta \bar{\eta} \sim \bar{\partial} X=0$. The space of holomorphic maps is finite dimensional and can be used as a basis for mathematical investigation. This leads to the connection between the topological string theory and the Gromov-Witten invariants, which we will discuss later. An example of $Q$ invariant amplitudes is $\mathcal{A}_{g}(n, m)$ given in (2.4) evaluated for a $Q$-closed state in $\mathcal{H}^{\otimes n} \otimes \mathcal{H}^{* \otimes m}$. Such amplitudes can be expressed as sums over holomorphic maps. On the other hand, $\omega_{g}(n, m)$ as in (2.6) is invariant under $D=d+Q$ but not under $Q$. This leads to an interesting subtlety in evaluating $F_{g}=\int_{\mathcal{M}_{g}(n, m)} \omega_{g}(n, m)$. In particular, it will show up as the holomorphic anomalies [12,10], as we will see below.

Let us turn to the B-model. Its degrees of freedom are maps $X: \Sigma \rightarrow M$ and Grassmannian fields on $\Sigma$,

$$
\begin{aligned}
& \bar{\theta}(z, \bar{z}) \in T_{X(z, \bar{z})}^{0,1} M, \quad \theta(z, \bar{z}) \in T_{X(z, \bar{z})}^{* 1,0} M, \\
& \eta(z, \bar{z}) \in\left(\Omega^{1,0}(\Sigma) \oplus \Omega^{0,1}(\Sigma)\right) \otimes T_{X(z, \bar{z})}^{1,0} M .
\end{aligned}
$$

The $Q$ transformation is given by

$$
\delta X^{i}=0, \quad \delta \bar{X}^{\bar{i}}=\epsilon \bar{\theta}^{\bar{i}} ; \quad \delta \eta^{i}=\epsilon d X^{i}, \quad \delta \theta=\delta \bar{\theta}=0
$$

The $Q$ cohomology of $\mathcal{H}$ in this case is the $\bar{\partial}$ cohomology,

$$
\mathrm{H}_{Q}(\mathcal{H})=\oplus_{p, q=0}^{\hat{c}} \mathrm{H}_{\bar{\partial}}^{p}\left(M, \wedge^{q} T^{1,0} M\right) .
$$

The degrees $(p, q)$ can be identified as eigenvalues of $\left(J_{0}, \bar{J}_{0}\right)$ in the left-moving and rightmoving $\mathcal{N}=2$ superconformal algebras. On a Calabi-Yau manifold of complex dimensions $\hat{c}$, there is a unique holomorphic $(\hat{c}, 0)$-form $\Omega$ which is nowhere vanishing, and it can be used to identify this cohomology with the Hodge-de Rham cohomology as,

$$
\mathrm{H}_{\bar{\partial}}^{p}\left(M, \wedge^{q} T^{1,0} M\right) \simeq \mathrm{H}^{p, \hat{c}-q}(M) .
$$

Fixed points of the $Q$ transformation (3.7) can be found at $d X=0$, namely constant maps $X: \Sigma \rightarrow p \in M$. Thus, $Q$ invariant amplitudes, such as conformal field theory amplitudes $\mathcal{A}_{g}(n, m)$ evaluated for $Q$ closed states in $\mathcal{H}^{\otimes n} \otimes \mathcal{H}^{* \otimes m}$, can be expressed as a sum over constant maps, which is the same thing as an integral over $M$. The string amplitudes $F_{g}(n, m)=\int_{\mathcal{M}_{g}(n, m)} \omega_{g}(n, m)$, on the other hand, does not necessarily localize to integrals over $M$ since they are invariant under $D=d+Q$ and not under $Q$. The difference of $D$ and $Q$ shows up as contributions from boundaries of the moduli space $\mathcal{M}_{g}(n, m)$, and the part of $F_{g}$ which fails to be localized on constant maps can be expressed in terms of Feynman diagrams for point particles. This is the origin of the Kodaira-Spencer description of the B-model mentioned at the end of section 2.2. 


\subsection{Moduli Space of Topological String Theory}

According to Yau's theorem, a Ricci-flat Kähler metric of a Calabi-Yau manifold $M$ is uniquely determined by complex structure and Kähler class. Infinitesimal deformations of complex structure correspond to elements of $\mathrm{H}_{\bar{\partial}}^{1}\left(M, T^{1,0} M\right)$, while Kähler class is parametrized by $H^{1,1}(M)$. In string theory, it is natural to complexify Kähler class. For each choice of complex structure and complexified Kähler class, there is a conformal field theory and we can use it to define string amplitudes $F_{g}(n, m)$. Thus, topological string amplitudes should be regarded as geometric objects over the moduli space of Calabi-Yau manifolds.

Infinitesimal deformations of a conformal field theory are generated by marginal operators, which in the case of a topologically twisted $\mathcal{N}=2$ conformal field theory correspond to $Q$ cohomology elements of $\left(J_{0}, \bar{J}_{0}\right)=(1,1)$. In the A and B-models, they are elements of $H^{1,1}(M)$ and $H_{\bar{\partial}}^{1}\left(M, T^{1,0} M\right)$ respectively, as we can see from (3.5) and (3.8). Thus, we expect that string amplitudes $F_{g}$ depend on the Kähler moduli in the A-model and the complex moduli in the B-model.

Due to the index theorem and the triviality of the first Chern class of $M$, the number of zero modes of fermions $\eta, \bar{\eta}$ minus the number of zero modes of $\theta, \bar{\theta}$ on $\Sigma_{g}$ is equal to $(2 g-2) \hat{c}$. When $\hat{c}=3$, this coincides with $\operatorname{dim} \mathcal{M}_{g}$. Because of this, $\omega_{g}$ with $n=m=0$ is

a top form on $\mathcal{M}_{g}$, and we can define the vacuum amplitude $F_{g}=\int_{\mathcal{M}_{g}} \omega_{g}$. We will mainly consider the case of $\hat{c}=3$ in the following, except for $g=1$, where the index vanishes. It turns out that this is also the most interesting case for physical applications of string theory since $2 \hat{c}=6=10-4$, where 10 is the critical dimensions of superstring theory and 4 is the macroscopic dimensions of our spacetime.

Let us discuss the moduli space of the topological string theory. It is easier to start with the B-model since its conformal field theory amplitudes are expressed as integrals over $M$ and we can use classical geometry to describe them. The moduli space of the B-model is the complex moduli space $\mathcal{M}_{C}$ of $M$. Since the holomorphic (3,0)-form $\Omega$ is unique up to scale, it defines a line bundle $\mathcal{L}$ over $\mathcal{M}_{C}$ (a sub-bundle of the Hodge bundle) with a natural metric,

$$
\|\Omega\|^{2}=i \int_{M} \Omega \wedge \bar{\Omega} .
$$

The metric on $\mathcal{M}_{C}$ is given as a curvature of this line bundle,

$$
G_{i \bar{j}}=\partial_{i} \bar{\partial}_{\bar{j}} K
$$


with the Kähler potential $K$,

$$
K=-\log \|\Omega\|^{2} .
$$

On $\mathcal{M}_{C}$, there are particularly useful coordinates called the flat coordinates. To define them, let us choose a symplectic basis of homology 3-cycles, $\left\{\alpha_{I}, \beta^{I}\right\}_{I=0,1, \cdots, h^{1,2}}$. Note that $\operatorname{dim} H_{3}=2+2 h^{1,2}$ since $h^{3,0}=1$ for a Calabi-Yau manifold. Let us consider the period integrals of $\Omega$,

$$
X^{I}=\int_{\alpha_{I}} \Omega, \quad F_{I}=\int_{\beta^{I}} \Omega .
$$

Since the complex structure of $M$ is determined by the periods $X^{I}$ over the $\alpha$ cycles, $F_{I}$ 's are functions of $X^{I}$ 's. Moreover, they are homogeneous functions of $X$ 's with weight 1 since $X^{I}$ 's and $F_{I}$ 's scale in the same way under scaling of $\Omega$. It is known that they can be expressed as derivatives of a single function $F(X)$ as,

$$
F_{I}(X)=\frac{\partial F(X)}{\partial X^{I}}
$$

where $F(X)$ is a homogeneous function of $X$ 's of weight 2. In physics literature, $F(X)$ is called the prepotential. Since scaling of $\Omega$ does not affect the complex structure, we can use ratios of $X$ 's as coordinates of the moduli space $\mathcal{M}_{C}$,

$$
t^{i}=\frac{X^{i}}{X^{0}}, \quad i=1, \cdots, h^{1,2}
$$

These $t$ 's are called the flat coordinates of $\mathcal{M}_{C}$ and play an important role in defining the mirror map from $M$ to its mirror partner. The prepotential $F(X)$ is not globally defined as a holomorphic section of $\mathcal{L}^{2}$ on the moduli space, because the periods $X^{I}, F_{I}$ undergo monodromy transformations. However, its third derivatives with respect to $t$ 's,

$$
C_{i j k}=\frac{\partial^{3} F}{\partial t^{i} \partial t^{j} \partial t^{k}}=\int_{M} \Omega \frac{\partial^{3} \Omega}{\partial t^{i} \partial t^{j} \partial t^{k}}
$$

define a global holomorphic section of $\mathcal{L}^{2} \otimes \operatorname{Sym}^{\otimes 3} T^{* 1,0} \mathcal{M}_{K}$. In physics literature, $C_{i j k}$ is called the Yukawa couplings. It is equal to the genus- 0 topological string amplitude $\mathcal{A}_{g=0}(3,0)$ evaluated for 3 elements of $\mathrm{H}^{1}\left(M, T^{0,1} M\right) \simeq \mathrm{H}^{1,2}(M)$ corresponding to the deformations $\partial_{i}, \partial_{j}, \partial_{k}$ of complex structure of $M$. Since the moduli space of a sphere with three punctures $\mathcal{M}_{g=0}(3,0)$ is 0 -dimensional, the string amplitude $\omega_{g=0}(3,0)$ is equal to the conformal field theory amplitude $\mathcal{A}_{g=0}(3,0)$. 
The existence of the line bundle $\mathcal{L}$ over the moduli space and its relation to the moduli space metric are general features of the topological string theory, not restricted to the B-model [13]. The $Q$ cohomology of $\mathcal{H}$ makes an analogue of the Hodge bundle over the moduli space and it can be used to define the metric and compute the genus- 0 string amplitudes. For more details, see Chapter 2 of [10].

In particular, the same structure exists for the A-model, even though we must move away from classical geometry in this case. Classically, the Kähler moduli space $\mathcal{M}_{K}$ is a cone in $H^{1,1}(M)$ where the metric in $M$ is positive definite and the Yukawa coupling is a constant tensor given by the intersection,

$$
C_{a b c}^{(\text {classical })}=\int_{M} u_{a} u_{b} u_{c},
$$

of $u_{a}, u_{b}, u_{c} \in \mathrm{H}^{1,1}(M)$. Quantum mechanically, the metric and the Yukawa coupling are corrected due to nontrivial holomorphic maps from $\Sigma_{g=0}$ to $M$. For example, the quantum corrected Yukawa coupling is given by

$$
C_{a b c}=\int_{M} u_{a} u_{b} u_{c}+\sum_{n} n_{a} n_{b} n_{c} N_{0, n} \frac{e^{2 \pi i n t}}{1-e^{2 \pi i n t}},
$$

where $N_{0, n}$ is the genus-0 Gromov-Witten invariants for holomorphic maps of degrees $n=\left(n_{1}, \cdots, n_{h^{1,1}}\right)$, and $t=\left(t^{1}, \cdots, t^{h^{1,1}}\right)$ are the flat coordinates of $\mathcal{M}_{K}$. In the A-model, t's are simply the linear coordinates on $\mathrm{H}^{1,1}(M)$. Once the Yukawa coupling is given, one can integrate $C_{a b c}=\partial_{a} \partial_{b} \partial_{c} F$ to compute the prepotantial $F$. The Kähler potential $K$ is then given by

$$
K=-\log \left(4 F-4 \bar{F}+\bar{t}^{a} \partial_{a} F-t^{a} \bar{\partial}_{\bar{a}} \bar{F}\right) .
$$

Due to the quantum corrections (3.18), the Kähler metric $G_{a \bar{b}}=\partial_{a} \bar{\partial}_{\bar{b}} K$ is not the one for the cone of $H^{1,1}(M)$ anymore, but it acquires more elaborate structure. In particular, Kähler moduli spaces of topologically distinct Calabi-Yau manifolds can be combined together into a single smooth moduli space [14,15].

A pair of Calabi-Yau manifolds $(M, \tilde{M})$ is called a mirror pair if the A-model for $M$ is equivalent to the B-model for $\tilde{M}$. It is an isomorphism of two conformal field theories. Thus, for example, the Hilbert space $\mathcal{H}$ of the A-model for $M$ is isomorphism to the Hilbert space $\tilde{\mathcal{H}}$ of the B-model for $\tilde{M}$, including how they are decomposed into irreducible representations of the $\mathcal{N}=2$ superconformal algebra. The first example of the mirror pair was found by B. Greene and M. R. Plesser [16]. The flat coordinate $t$ that appear in (3.18) 
in the A-model are ratios of the periods of $\Omega$ in the B-model and the Yukawa coupling (3.16) is also expressed in terms of the periods. Therefore, one can compute the genus-0 Gromov-Witten invariants of $M$ by evaluating the period integrals in $\tilde{M}$. This procedure was applied by P. Candelas, X. De La Ossa, P. Green, and L. Parkes [17] to the mirror pair of Greene and Plesser. It would be fair to say that it was their computation that demonstrated the power of the mirror symmetry for the first time and sparked interests in the mathematical community.

\section{Topological String at Higher Genera}

At genus-0, the A-model computes the number of holomorphic maps from a sphere to the Calabi-Yau manifold $M$, and the B-model amplitudes are given by the periods of the holomorphic $(3,0)$-form. The mirror symmetry relates these two computations. Since the mirror symmetry is an isomorphism of two conformal field theories, we expect that the relation

$$
F_{g}^{\mathrm{A}-\operatorname{model}}(M)=F_{g}^{\mathrm{B}-\operatorname{model}}(\tilde{M}) .
$$

continues to hold for $g \geq 1$. We note that $F_{g}$ is a section of $\mathcal{L}^{2-2 g}$.

\subsection{Genus One}

The genus- 1 case is special for two reasons [12]. Firstly, we do not have to restrict to the case of $\hat{c}=3$ to consider the vacuum amplitude $F_{g}$. This is because, for $g=1$, the index theorem mentioned in the last section only says that $\eta$ and $\theta$ have the same number of zero modes for $g=1$, and this does not prevent $\omega_{g=1}(0,0)$ from carrying a top form on $\mathcal{M}_{g=1}$ for any $\hat{c}$. Secondly, since $\Omega^{0,1}(\Sigma)$ and $\Omega^{1,0}(\Sigma)$ are trivial on a genus-1 surface, the A-model (3.3) and the B-model (3.6) have the same set of fields. What happens is that the genus-1 vacuum amplitude is a sum of two contributions, one depends on the Kähler moduli $(t, \bar{t})$ and another depends on the complex moduli $(\tau, \bar{\tau})$ of $M$,

$$
F_{1}=F_{1}^{\mathrm{A}-\text { model }}(t, \bar{t})+F_{1}^{\mathrm{B}-\text { model }}(\tau, \bar{\tau}) .
$$

It is instructive to consider the case when $M$ is an elliptic curve $T^{2}$. We use the standard parameter $\tau$ for the complex moduli of $T^{2}$. The Kähler moduli $t$ can be chosen so that its imaginary part is the area of $T^{2}$. In this case, the conformal field theory on $\Sigma$ consists of a complex-valued massless free scalar field (corresponding to the map 
$X: \Sigma \rightarrow T^{2}$ ) and a few massless free fermions. These fields are free since the metric on $T^{2}$ is flat. This means that their path integrals are Gaussian with coefficients given by the Laplacians on $\Sigma$. Thus the conformal field theory amplitude $\omega_{g=1}$ is given by a sum over harmonic maps from $\Sigma$ to $T^{2}$ weighted by the determinants of the Laplacians on $X$ and on $\eta, \theta$. The determinants cancel out due to the $Q$ invariance, and the sum over harmonic maps and the integral of the resulting $\omega_{g=1}$ over the moduli space $\mathcal{M}_{g=1}$ can be carried out using the Poisson re-summation method. The result is

$$
F_{1}=-\log \left(\sqrt{\operatorname{Im} t}|\eta(t)|^{2}\right)-\log \left(\sqrt{\operatorname{Im} \tau}|\eta(\tau)|^{2}\right)
$$

where $\eta(\tau)$ is the Dedekind eta-function. We note that the genus-1 amplitude is expressed as a sum of the $t$ dependent term and the $\tau$ dependent term, as expected in (4.2). On the other hand, the holomorphic splitting is spoiled by the holomorphic anomalies,

$$
\frac{\partial^{2} F_{1}}{\partial t \partial \bar{t}}=\frac{1}{2(\operatorname{Im} t)^{2}}, \quad \frac{\partial^{2} F_{1}}{\partial \tau \partial \bar{\tau}}=\frac{1}{2(\operatorname{Im} \tau)^{2}} .
$$

The A-model part of $F_{1}$ counts the number of holomorphic maps in an appropriate sense. To see this, it is useful to write

$$
\frac{i}{2 \pi}{\frac{\partial F_{1}}{\partial t}}_{\mid \bar{t} \rightarrow \infty}=\frac{1}{24}-\frac{1}{2} \sum_{M} e^{2 \pi i|\operatorname{det} M| t},
$$

where the sum in the right-hand side is over $M \in G L(2, Z)$ such that it maps $\tau$ into the fundamental domain of $\mathcal{M}_{g=1}$. This can be interpreted as a sum over holomorphic maps from $\Sigma$ to $T^{2}$. For a generic complex structure of $\Sigma$, there is no holomorphic map from $\Sigma$ to $T^{2}$. Thus, the counting of holomorphic maps makes sense only when we integrate over $\mathcal{M}_{g=1}$. The complex moduli dependent part $-\log \left(\sqrt{\operatorname{Im} \tau}|\eta(\tau)|^{2}\right)$ has the familiar expression as the logarithm of the determinant of the Laplacian on $T^{2}$. It is worthwhile to note that the torus $T^{2}$ is self-mirror. Namely, the mirror of $T^{2}$ is another $T^{2}$ with the Kähler moduli and the complex moduli exchanged. The exchange symmetry of (4.3) under $t \leftrightarrow \tau$ is a consequence of the mirror symmetry in this case.

For a general Calabi-Yau manifold $M$, the splitting (4.2) continues to hold. The A-model amplitude $F_{1}^{\mathrm{A}-\text { model }}$ computes the genus-1 Gromov-Witten invariants, 3

$$
\begin{aligned}
\frac{i}{2 \pi} \frac{\partial F_{1}^{\mathrm{A}-\text { model }}}{\partial t^{a}} \mid \bar{t} \rightarrow \infty & =\frac{(-1)^{\hat{c}}}{24} \int_{M} u_{a} \wedge c_{\hat{c}-1}-\sum_{n} n_{a} N_{1, n} \sum_{m} \frac{m e^{2 \pi i m n t}}{1-e^{2 \pi i m n t}} \\
& -\frac{1}{12} \sum_{n} n_{a} N_{0, n} \frac{e^{2 \pi i n t}}{1-e^{2 \pi i n t}},
\end{aligned}
$$

3 Note that some of the expressions for $F_{1}$ given in [12] are missing a factor of $1 / 2$ since we did not properly take into account the $Z_{2}$ automorphism of $T^{2}$. This has been corrected in [10]. 
where $c_{n}$ is the $n$-th Chern-Class of $M$, and $u_{a} \in H^{1,1}(M)$ corresponds to the deformation of the Kähler moduli $\partial / \partial t^{a}$. Note the contributions from the genus-0 Gromov-Witten invariants in the second line in the right-hand side. A mathematical explanation of these contributions is given by S. Katz in the appendix to [12].

The B-model amplitude $F_{1}^{\mathrm{B}-\text { model }}$ can be expressed in terms of determinants of delbar operators on various bundles over $M$. Let $V$ be a holomorphic vector bundle over $M$ and $\Delta_{V}^{(p)}=\bar{\partial}_{V}^{\dagger} \bar{\partial}_{V}$ on $\Omega^{0, p}(M) \otimes V$. The holomorphic Ray-Sinter torsion for $V$ is defined by

$$
I(V)=\prod_{p}\left(\operatorname{det}^{\prime} \Delta_{V}^{(p)}\right)^{(-1)^{p} p} .
$$

It is important to note that $I\left(V_{1}\right) / I\left(V_{2}\right)$ is independent of the Kähler structure of $M$ [18]. The genus-1 B-model amplitude is given by their combination as

$$
F_{1}^{\mathrm{B}-\text { model }}=\frac{1}{2} \sum_{q}(-1)^{q} q \log I\left(\Omega^{q, 0}\right) .
$$

In [12], the holomorphic anomaly equation for $F_{1}$ was derived using the invariance of $\omega_{g=1}$ under $D=d+Q$. The idea is to use the invariance to relate the $Q$ trivial operation (such as $\partial^{2} / \partial t \partial \bar{t}$ ) on $F_{1}$ to contributions from the boundary of $\mathcal{M}_{g=1}$. The result, for a Calabi-Yau threefold $M$, is given by

$$
\partial_{i} \bar{\partial}_{\bar{j}} F_{1}=\frac{1}{2} C_{i m n} \bar{C}_{\bar{j} \bar{m} \bar{n}} e^{2 K} G^{m \bar{m}} G^{n \bar{n}}-\left(\frac{\chi(M)}{24}-1\right) G_{i \bar{j}}
$$

where $\chi(M)$ is the Euler characteristic of $M$. In sections 5.6 - 5.8 of [10], it was shown that this agrees with the application of the Quillen anomaly formula,

$$
\partial \bar{\partial} \log I(V)=\partial \bar{\partial} \sum_{p}(-1)^{p} d_{p}+2 \pi i \int_{M} \operatorname{Td}(T M) \operatorname{Ch}(V),
$$

where $d_{p}$ is the determinant of the inner product in the kernel of $\bar{\partial}_{V}$ on $\Omega^{0, p}(M) \wedge V$, Td is the Todd class and $\mathrm{Ch}$ is the Chern class. However, the (4.9) does not assume that $F_{1}$ is given in terms of the Ray-Singer torsions. In particular, it applies to the A-model also.

The mirror symmetry (4.1) for the genus-1 amplitudes relates the genus-1 GromovWitten invariants in (4.6) to the Ray-Singer torsions (4.8). In [12], this was used to compute $N_{g=1, n}$ explicitly for the quintic threefold. The result was recently proven mathematically by A. Zinger [19]. The result of [12] also predicted a certain behavior of the torsions (4.8) at boundaries of the moduli space of the mirror of the quintic threefold. This prediction was also proven recently by H. Fang, Z. Lu, and K. Yoshikawa [20]. 


\subsection{Genus greater than One}

The A-model amplitude $F_{g}^{\mathrm{A}-\text { model }}$ for $g>1$ is related to the genus- $g$ Gromov-Witten invariants as (sections $5.10-5.13$ of $[10$ ),

$$
\begin{aligned}
F_{g \mid \bar{t} \rightarrow \infty}^{\mathrm{A}-\text { model }}= & \frac{1}{2} \chi(M) \int_{\mathcal{M}_{g}} c_{g-1}^{3}+\sum_{n} N_{g, n} e^{2 \pi i n t} \\
& +\left(\text { contributions from } N_{g^{\prime}<g, n} \text { and multicoverings }\right),
\end{aligned}
$$

where $c_{n}$ is the $n$-th Chern class of the Hodge bundle over $\mathcal{M}_{g}$. The formula for the first term in the right-hand side was derived in 21] as

$$
\int_{\mathcal{M}_{g}} c_{g-1}^{3}=\frac{(-1)^{g-1}}{(2 \pi)^{2 g-2}} 2 \zeta(2 g-2) \chi_{g},
$$

where $\chi_{g}$ is the Euler characteristics of $\mathcal{M}_{g}$ given by

$$
\chi_{g}=\frac{(-1)^{g-1}}{2 g(2 g-2)} B_{g}
$$

The B-model amplitude is formally expressed as a sum of Feynman diagrams for quantization of the Kodaira-Spencer theory (sections 5.1-5.4 of [10]). Although such an expression is a natural generalization of the genus-one result relating $F_{1}$ to the Ray-Singer torsion - in quantum field theory, one-loop amplitudes are given by determinant of Laplace operators - not much is known on how to carry out the computation beyond $g=1$ using Feynman diagrams.

The holomorphic anomaly equation found in [10] has turn out to be a useful tool to compute $F_{g}$ for higher genera,

$$
\bar{\partial}_{\bar{i}} F_{g}=\frac{1}{2} \bar{C}_{\bar{i} \bar{j} \bar{k}} e^{2 K} G^{j \bar{j}} G^{k \bar{k}}\left(D_{j} D_{k} F_{g-1}+\sum_{r=1}^{g-1} D_{j} F_{r} D_{k} F_{g-r}\right) .
$$

It is the only known method to compute $F_{g}$ systematically for higher genera in the case of compact Calabi-Yau manifolds. In [10], a diagramatic method was developed to obtain a general solution to the holomorphic anomaly equation recursively in $g$, to all order in g. Recently, the method was made more efficient by S. Yamaguchi and S.-T. Yau [22]. In [23], it was shown that, using known behavior of $F_{g}$ at boundaries of the Calabi-Yau moduli space, the holomorphic anomaly equations can be integrated to obtain $F_{g}$ up to $g=51$ for the quintic threefold. It is hoped that, with a better understanding of boundary data, we can go to even higher genera. In fact, the asymptotic behavior of $F_{g}$ for $g \rightarrow \infty$ for compact Calabi-Yau manifolds is needed to clarify the relation between the topological string theory and quantum states of black holes to be discussed later. 


\section{Open/Closed String Duality}

In section 2.2, we defined open string theory for a given choice of boundary conditions $\left\{\mathcal{E}_{i}: i=1, \cdots, k\right\}$. Let us denote the topological string amplitude of genus- $g$ with $n_{i}$ boundaries of type $\mathcal{E}_{i}(i=1, \cdots, k)$ by $F_{g ; n_{1}, \ldots, n_{k}}$. It is useful to consider a generating function with weights $t_{1}, \cdots, t_{k}$ as,

$$
F_{g}(t)=\sum_{n_{1}, \cdots, n_{k}} F_{g ; n_{1}, \ldots, n_{k}} t_{1}^{n_{1}} \cdots t_{k}^{n_{k}}
$$

The open/closed string duality is a conjecture that there is a family $\tilde{M}(t)$ of a CalabiYau threefold such that the generating function $F_{g}(t)$ defined in the above is the genus- $g$ vacuum amplitude for closed topological string on $\tilde{M}(t)$. The idea of open/closed string duality originally appeared in the paper by G. 't Hooft [24] 24 years ago. For this reason, $t_{i}$ 's are called 't Hooft couplings.

Let us present a couple of examples for this. The vacuum amplitude of the ChernSimons gauge theory on $S^{3}$ is given by 25]

$$
Z\left(S^{3}\right)=\frac{e^{i \frac{\pi}{8} N(N-1)}}{(k+N)^{\frac{N}{2}}} \sqrt{\frac{k+N}{N}} \prod_{s=1}^{N-1}\left[2 \sin \left(\frac{s \pi}{k+N}\right)\right]^{N-s}
$$

Here $k$ is the level of the Chern-Simons theory and the gauge group is $U(N)$. In [8], it was observed that $Z\left(S^{3}\right)$ can be expressed as

$$
Z\left(S^{3}\right)=\exp \left(-\sum_{g, n} F_{g, n} \lambda^{2 g-2} t^{n}\right)
$$

where the string coupling $\lambda$ and the 't Hooft coupling $t$ are given in terms of the ChernSimons variables as

$$
\lambda=\frac{2 \pi}{k+N}, \quad t=i \lambda N
$$

and that $F_{g, n}$ is the A-model amplitude of genus $g$ with $n$ boundaries. The target space is the cotangent space of $S^{3}$, which is a non-compact Calabi-Yau threefold, and the boundary condition is set so that open strings end on the base $S^{3}$ of $T^{*} S^{3}$, or more specifically the map $X: \Sigma_{g, n} \rightarrow T^{*} S^{3}$ obeys the Neumann condition along $S^{3}$ and the Dirichlet condition transverse to $S^{3}$. The boundary conditions for the fermions $\eta, \theta$ are determined so that the $Q$ symmetry is preserved. The fact that the base $S^{3}$ is a Lagrangian sub-manifold of 
$T^{*} S^{3}$ then guarantees the $Q$ symmetry. Physicists refer to this boundary condition as "D branes are wrapping the base $S^{3}$ of $T^{*} S^{3}$."

Subsequently, Gopakumar and Vafa pointed out that the generating function of $F_{g, n}$ with the 't Hooft coupling $t$ can be expressed as [26]

$$
\begin{aligned}
F_{g}(t) & =\sum_{n} F_{g, n} t^{n} \\
& =\int_{\mathcal{M}_{g}} c_{g-1}^{3}-\frac{\chi_{g}}{(2 g-3) !} \sum_{n=1} n^{2 g-3} e^{2 \pi i n t},
\end{aligned}
$$

where the Hodge integral and the Euler characteristics $\chi_{g}$ in the right-hand side are given in the last section in (4.12) and (4.13). This is exactly equal to the genus-g A-model amplitude for the small resolution of the conifold $u^{2}+v^{2}+x^{2}+y^{2}=0$, where we can identify $t$ as the Kähler moduli for the blown up $P^{1}$. Thus, they conjectured that the open string theory on $T^{*} S^{3}$ is equivalent to the closed string theory on the resolved conifold, where the 't Hooft coupling $t$ in the open string side is identified with the amount of blow up on the closed string side.

Since the Chern-Simons gauge theory can be used to compute invariants of knots and links in three dimensions [25], it is natural to ask if there are closed string duals for such computations also. To compute these invariants in the topological string theory, one needs to introduce another D brane (i.e. another Lagrangian sub-manifold of $T^{*} S^{3}$ ) which intersects with the base $S^{3}$ along the knot or link in question. One can take the closed string dual of this set up and find that the same knot invariants can be computed in the resolved conifold [27]. This result predicted new algebraic structure of knot and link invariants and the existence of new integer invariants, which were proven in [28].

In this way, knot invariants can be regarded as topological string amplitudes with 2 types of $\mathrm{D}$ branes; one is the base $S^{3}$ of $T^{*} S^{3}$ and another is determined by the choice of knot. A generalization of this for 3 types of $\mathrm{D}$ branes gives the topological vertex, which can be used to compute $F_{g}$ for any toric Calabi-Yau manifold [29]. For more detail, see [30].

So far we have been discussing open topological string associated to the A-model. According to [8], an analogue of the Chern-Simons gauge theory in the B-model is the holomorphic Chern-Simons theory. A particularly simple case is when $\mathrm{D}$ branes wrap $P^{1}$ in a Calabi-Yau space. Consider the singular space,

$$
u^{2}+v^{2}+y^{2}+W^{\prime}(x)^{2}=0 \text { in } C^{4},
$$


where $W(x)$ is a degree $(k+1)$ polynomial $x^{k+1}+\cdots$. We can make small resolution and blow up $k P^{1}$ 's. We can then consider $k$ different boundary conditions, one for each $P^{1}$. It was shown in [9] that $F_{g ; n_{1}, \cdots, n_{k}}$ in this case is computable by the random matrix model with a potential given by $\operatorname{tr} W(M)$,

$$
\int d M \exp \left[-\frac{1}{\lambda} \operatorname{tr} W(M)\right]=\exp \left[-\sum_{g, n_{1}, \cdots, n_{k}} F_{g ; n_{1}, \cdots, n_{k}} \lambda^{2 g-2} t_{1}^{n_{1}} \cdots t_{k}^{n_{k}}\right]
$$

where $\int d M$ is an integral over $N \times N$ matrices, $t_{i}=i \lambda N_{i}$, and $N_{i}$ is the number of eigenvalues of $M$ near the $i$-th critical point of $W(x)$. The closed string dual is the topological string on the deformed geometry,

$$
u^{2}+v^{2}+y^{2}+W^{\prime}(x)^{2}+f(x)=0, \quad(u, v, x, y) \in C^{4},
$$

for some polynomial $f(x)$ determined by $t_{i}$ 's.

It is important to note that the open/closed string duality holds for each genus separately. As such, it is a property of two-dimensional conformal field theories. In [31], a microscopic derivation of the Gopakumar-Vafa duality was given by studying phases of the conformal field theory. It is hoped that the AdS/CFT correspondence [32, 33] can be derived in a similar way.

\section{Quantum States of Black Holes}

So far we have discussed mainly mathematical aspects of topological string theory. Indeed, in the earlier days, the topological string theory was studied as a toy model of string theory. It is worth pointing out, however, that Witten already expressed the vision in his pioneering paper [34] that the topological string theory may describe a new phase of string theory where general covariance is unbroken. 1 By the early 90's, it was also realized that the genus-0 topological string amplitude $F_{0}(M)$ can be used for some computation in physical superstring compactified on $M$ times the four-dimensional Minkowski space. The third derivatives of $F_{0}$ given by (3.16) and (3.18) are called the Yukawa couplings since they are related to the couplings of two spinors to one scalar field in the heterotic string compactified on a Calabi-Yau manifold [35]. See also section 6 of [11].

4 In the Minkowski space, general covariance is spontaneously broken to the Poincare symmetry. 
In [10], the relation to topological string and physical string was generalized for all $g \geq$ 1. It turned out that a certain series of higher derivative terms in the low energy effective action of type IIA / IIB superstring theory compactified on $M$ times the Minkowski space is computed by $F_{g}$ 's of the A / B-model. But, what are these terms in the low energy effective theory good for? One answer to this question was found in black holes.

Black holes are classical solutions to the Einstein equation coupled to matter fields. There are singularities, but they are covered by event horizons. Anything that goes inside of the horizons will not be able to come back out. As classical solutions, black holes are characterized by a very small set of parameters such as mass, angular momentum, and electric and magnetic charges. In contrast, a quantum black hole is expected to carry a large number of quantum states, roughly the exponential of the area of the horizon measured in the unit of the Planck area $\sim 10^{-70} \mathrm{~m}^{2}$. For example, a typical astrophysical black hole of mass $\sim 10^{31} \mathrm{~kg}$ would have about $e^{10^{78}}$ states. This expectation is based on the following.

For classical solutions to the Einstein equation with an energy-momentum tensor satisfying the positive energy condition, S. Hawking proved the black hole area theorem stating that the total area of black hole event horizons cannot decrease in any classical process [36]. Based on an analogy between this theorem and the second law of thermodynamics, which states that the thermodynamic entropy can only increase in time, J. Bekenstein proposed that a black hole carries an entropy $S$ proportional to the area $A$ of its horizon [37]. The proportionality constant was undetermined at that time, however. Within a year, S. Hawking [38] used the semi-classical quantization of spacetime geometry and matter near the horizon and showed that a black hole of mass $M$ emits the black body radiation with temperature $T$ consistent with the standard thermodynamic formula

$$
\frac{1}{T}=\frac{\partial S}{\partial M}
$$

if the entropy $S$ is given by

$$
S=\frac{1}{4} \frac{A}{\ell_{\mathrm{P}}^{2}}
$$

where $\ell_{\mathrm{P}} \sim 1.6 \times 10^{-35} \mathrm{~m}$ is the Planck length. Hawking's computation gave an evidence for Bekenstein's conjecture and also fixed the proportionality constant to be $\left(4 \ell_{\mathrm{P}}^{2}\right)^{-1}$. Since the area $A$ of the horizon scales as $M^{2}$, this entropy formula predicts that a black hole of mass $M$ should have quantum states as many as $\sim e^{\left(\frac{M}{M_{\mathrm{P}}}\right)^{2}}$, where the Planck mass $M_{\mathrm{P}} \sim$ $10^{-8} \mathrm{~kg}$. The number $e^{10^{78}}$ quoted in the previous paragraph is obtained by estimating 
$e^{S}$ for astrophysical black holes. The proposed entropy formula (6.2) can be compared to the definition of the thermodynamic entropy by R. Clausius in the 19th century. The Bekenstein-Hawking formula (6.2) was proposed based on macroscopic properties of black holes, and it calls for a microscopic explanation just as Boltzmann recognized that the thermodynamic entropy is the logarithm of the number of states and explained much of classical thermodynamics based on the microscopic point of view. Doing the same for the black hole entropy was posed as a challenge to any theory that claims to unify quantum mechanics and general relativity consistently.

String theory has partially met this challenge. There is a class of black holes in superstring theory called BPS black holes. These solutions are invariant under non-trivial subalgebras of supersymmetry, and their Hawking temperatures are zero. These black holes are therefore stable, and their quantum states can be reliably counted. Thanks to the D brane construction by Polchinski [39], some of them can be realized in terms of D branes in superstring theory. This description is useful since low energy quantum states of these black holes can be described using supersymmetric gauge theories on D branes. A. Strominger and C. Vafa used this technique to count the number of quantum states of BPS black holes and found a perfect agreement with the prediction of Bekenstein and Hawking in the limit when the masses of black holes are asymptotically large [40. One has to take this limit since Hawking's computation in 38 is reliable only in this limit. As the black hole becomes small, the curvature near the horizon becomes strong and the semi-classical approximation breaks down.

To deepen our understanding of the black hole microstates, we need to know what happens for small black holes. In fact, the counting of microstates typically becomes simpler for small black holes since the gauge theory on D branes becomes weakly coupled. On the other hand, the gravity side of the story becomes more complicated. The strong curvature at the horizon means that we must take into account corrections to Einstein's theory due to stringy effects and quantum gravity effects. Remarkably, topological string amplitudes $F_{g}$ compute exactly such corrections for small BPS black holes. Inspired by the earlier work [41], the following conjecture was formulated in [42].

For definiteness, let us consider type IIB superstring theory compactified on a CalabiYau manifold. For type IIA, we take the mirror of the story below. Let us choose the symplectic basis of homology 3 -cycles, $\left\{\alpha_{I}, \beta^{I}\right\}_{I=0,1, \cdots, h^{1,2}}$ on $M$. In type IIB string, there are D3 branes - it means that we can consider boundary conditions such that open strings end on a $(3+1)$ dimensional submanifold in the four-dimensional Minkowski space times 
$M$. Submanifols are chosen to be special Lagrangian submanifolds of $M$, as required by supersymmetry [43,44, times a time-like direction in the Minkowski space. Seen in four dimensions, it is a particle moving in the time-like direction. We can consider a bound state of several D branes. Take a bound state of D3 branes whose total homology class is given by $\sum_{I} p^{I} \alpha_{I}+q_{I} \beta^{I}$ for some integers $\left(p^{I}, q_{I}\right)$. Seen in four dimensions, the mass of the resulting particle is proportional to the total volume of the special Lagrangian submanifolds. Clearly, the mass of the particle becomes large as $\left(p^{I}, q_{I}\right)$ increases. As the mass becomes large, the particle starts influencing the spacetime around it. In the limit of large mass, the Schwarzschild radius becomes longer than the Compton wave-length, and the geometry is described by BPS black hole solutions of the type discussed in the previous paragraph. What we want to compute is the number of quantum states of D3 branes for arbitrary values of $\left(p^{I}, q_{I}\right)$. Let us call the number $\sigma(p, q)$.

To state the conjecture on $\sigma(p, q)$, we need to set up some notation for topological string amplitudes $F_{g}$. In the B-model, $F_{g}$ is a section of $\mathcal{L}^{2-2 g}$ over the moduli space of complex structure of $M$. The complex structure can be parametrized by the periods $X^{I}=\int_{\alpha_{I}} \Omega$ of the holomorphic (3,0)-form. Since $\mathcal{L}$ is the sub-bundle of the Hodge bundle associated to scaling of $\Omega$, we can regard $F_{g}$ as a homogeneous function of $X^{I}$ 's of weight $(2-2 g)$.

The conjecture of [42] is that

$$
\sum_{q_{I}} \sigma(p, q) e^{-\pi \phi^{I} q_{I}}=|\exp (-F(X))|^{2}
$$

where $F(X)$ is the sum of the topological string amplitudes to all genera,

$$
F(X)=\sum_{g=0}^{\infty} F_{g}(X)
$$

and the periods $X^{I}$ are fixed as

$$
X^{I}=p^{I}+i \phi^{I}
$$

Since $F_{g}(X)$ is a homogeneous function of $X$ of weight $(2-2 g)$, we should think of $F(X)=$ $\sum_{g=0}^{\infty} F_{g}(X)$ in (6.4) as an asymptotic expansion for large $X$. If we neglect the higher genus terms $F_{g \geq 1}(X)$ in (6.4) and simply use the leading term $F_{0}(X)$ for $F(X)$ in (6.3), we reproduce the Bekenstein-Hawking formula (6.2) by $S=\log \sigma(p, q)$ evaluated for large $(p, q)$. Thus, the conjecture is that the subleading terms (6.4) - terms with $g \geq 1$ represent quantum gravity corrections to the entropy formula. 
The gauge theory computation of $\sigma(p, q)$ takes a familiar form in type IIA superstring theory. In this case, instead of D3 branes wrapping 3 cycles in $M$, we should consider D0, D2, D4 and D6 branes on holomorphic cycles in $M$. In particular, D0 branes are points and D6 branes wraps the entire Calabi-Yau manifold. By the homological mirror symmetry of Kontsevich, $p^{I}$ count the numbers of D4 and D6 branes, and $q_{I}$ count the numbers of D0 and D2 branes. If there are no D6 branes, the gauge theory on D4 branes is the $\mathcal{N}=4$ supersymmetric gauge theory topologically twisted and the number of quantum states is computed by the Witten index as in the work by Vafa and Witten [45. It is the Euler characteristics of the instanton moduli space. The numbers of D0 and D2 branes are the second and the first Chern classes of the gauge bundles on D4 branes. The left-hand side of (6.3) then becomes the generating function of the Euler characteristics of the instanton moduli space on D4 branes of the type studied by H. Nakajima [46]. With D6 branes, one has to think about a gauge theory in six dimensions.

Therefore, in mathematical terms, the conjectured formula (6.3) relates the GromovWitten invariants of $M$ to the Euler characteristics of the moduli space of instantons on four manifolds embedded in $M$, in the case of the A-model. For some non-compact Calabi-Yau manifolds, both sides of the formula can be evaluated explicitly, resulting in non-trivial checks of the conjecture [47,48]. Though the formula (6.3) has not been proven even to the standard of physicists, there have been several promising attempts and they have deepened our understanding of topological string, black holes microstates, and the AdS/CFT correspondence [49,50,51,52,53, 54,55]. To understand (6.3) better, it appears useful to develop a method to estimate of $F_{g}$ for large $g$ for compact Calabi-Yau manifolds.

\section{Concluding Remarks}

There are many aspects of the topological string theory I could not cover in this set of lectures. In particular, the topological vertex [29] to compute $F_{g}$ for toric Calabi-Yau manifolds is only briefly mentioned in section 5. Among other important discoveries I could not mention in these lectures are the relations of the topological string theory to dimer models and crystal melting [56], to the Donaldson-Thomas theory [57,58,59], to the Seiberg-Witten theory [60], and to BPS counting in M theory [61,62]. More recently, the non-commutative version of the Donaldson-Thomas theory has been formulated in [63, 64] and its relation to the crystal melting model and the black hole microstate counting have been pointed out in 65] generalizing the result of [56]. 
Though the topological string theory has rich mathematical structure and a broad range of applications to problems in physics, one should be reminded that it is still a simplified version of superstring theory. There is a vast terra incognita in superstring theory, and we need new mathematics to guide us through this territory. In this connection, it is also humbling to note that, after 80 years since the conception of quantum field theory by Heisenberg and Pauli [66], there has been no proof that it exists as a consistent mathematical theory, except for some cases in lower dimensions. In fact, the existence proof of quantum version of the Yang-Mills theory in four dimensions with a demonstration of its confinement property is posed as one of the Seven Millennium Problems by the Clay Mathematics Institute.

After the discovery of D brane construction by Polchinski and the AdS/CFT correspondence by Maldacena, many quantum field theories have been found to be dual (i.e., equivalent) to string theory in various geometries. This duality between quantum field theory and string theory has been used to evaluate non-perturbative effects in quantum field theories that are not accessible in any other method. At the same time, many fundamental issues in string theory have been translated into quantum field theory questions and this has shed important lights on mysteries of quantum gravity such as the black hole information paradox of Hawking. Because of this development, research in quantum field theories has become increasingly intertwined with string theory research. The progress in the past 10 years suggests that an ultimate solution to the Yang-Mills Problem could be built on a foundation where quantum field theories and string theory are extended and transformed into a single mathematical framework.

\section{Acknowledgments}

I would like to thank the organizing committee of the Takagi Lectures for inviting me to present these lectures and for their hospitality in Kyoto. I would like to thank collaborators on my works described in these lectures, especially M. Bershadsky, S. Cecotti, A. Strominger, and C.Vafa.

This work is supported in part by DOE grant DE-FG03-92-ER40701, by a Grantin-Aid for Scientific Research (C) 20540256 from the Japan Society for the Promotion of Science, by the World Premier International Research Center Initiative of MEXT of Japan, and by the Kavli Foundation. 


\section{References}

[1] T. Takagi, "Über eine Theorie des relativ Abel'schen Zahlkörpers," J. of the College of Science, Imperial Univ. of Tokyo 41-0, 1 (1920)

[2] T. Takagi, "Kinsei Suugaku Shidan," (Iwanami, 1995).

[3] T. Takagi, "Kaiseki Gairon," (Iwanami, 1983).

[4] G. Segal, "Two-dimensional conformal field theories and modular functors," IXth International Congress on Mathematical Physics (Swansea, 1988), pp. 22 - 37.

[5] G. Segal, "The definition of conformal field theory," in Topology, geometry and quantum field theory, London Math. Soc. Lecture Note Ser., pp. 421 - 577, First circulated in 1988.

[6] E. Witten, "Chern-Simons gauge theory as a string theory," Prog. Math. 133, 637 (1995) arXiv:hep-th/9207094.

[7] E. Witten, "Noncommutative geometry and string field theory," Nucl. Phys. B 268, 253 (1986).

[8] E. Witten, "Chern-Simons gauge theory as a string theory," Prog. Math. 133, 637 (1995) arXiv:hep-th/9207094.

[9] R. Dijkgraaf and C. Vafa, "Matrix models, topological strings, and supersymmetric gauge theories," Nucl. Phys. B 644, 3 (2002) arXiv:hep-th/0206255; "On geometry and matrix models," Nucl. Phys. B 644, 21 (2002) arXiv:hep-th/0207106].

[10] M. Bershadsky, S. Cecotti, H. Ooguri and C. Vafa, "Kodaira-Spencer theory of gravity and exact results for quantum string amplitudes," Commun. Math. Phys. 165, 311 (1994) arXiv:hep-th/9309140.

[11] E. Witten, "Mirror manifolds and topological field theory," in Mirror Symmetry I, ed. S.-T. Yau, pp. 121 - 160 (American Mathematica Society, 1998) arXiv:hepth/9112056].

[12] M. Bershadsky, S. Cecotti, H. Ooguri and C. Vafa, "Holomorphic anomalies in topological field theories," Nucl. Phys. B 405, 279 (1993) arXiv:hep-th/9302103.

[13] S. Cecotti and C. Vafa, "Topological antitopological fusion," Nucl. Phys. B 367, 359 (1991).

[14] E. Witten, "Phases of $\mathcal{N}=2$ theories in two dimensions," Nucl. Phys. B 403, 159 (1993) arXiv:hep-th/9301042.

[15] P. S. Aspinwall, B. R. Greene and D. R. Morrison, "Multiple mirror manifolds and topology change in string theory," Phys. Lett. B 303, 249 (1993) arXiv:hepth/9301043]; "Calabi-Yau moduli space, mirror manifolds and spacetime topology change in string theory," Nucl. Phys. B 416, 414 (1994) arXiv:hep-th/9309097.

[16] B. R. Greene and M. R. Plesser, "Duality in Calabi-Yau moduli space," Nucl. Phys. B 338, 15 (1990). 
[17] P. Candelas, X. C. De La Ossa, P. S. Green and L. Parkes, "A pair of Calabi-Yau manifolds as an exactly soluble superconformal theory," Nucl. Phys. B 359, 21 (1991).

[18] D. B. Ray and I. M. Singer, "Analytic torsion for complex manifolds," Ann. Math. 98, 54 (1973).

[19] A. Zinger, "The reduced genus-one Gromov-Witten invariants of Calabi-Yau hypersurfaces," [math/0705.2397].

[20] H. Fang, Z. Lu, and K. Yoshikawa, "Analytic torsion for Calabi-Yau threefolds," [math.DG/060141].

[21] C. Faber and R. Pandharipande, "Hodge integrals and Gromov-Witten theory," math.AG/9810173.

[22] S. Yamaguchi and S. T. Yau, "Topological string partition functions as polynomials," JHEP 0407, 047 (2004) arXiv:hep-th/0406078.

[23] M.-X. Huang, A. Klemm and S. Quackenbush, "Topological string theory on compact Calabi-Yau: modularity and boundary conditions," arXiv:hep-th/0612125.

[24] G. 't Hooft, "A planar diagram theory for strong interactions," Nucl. Phys. B 72, 461 (1974).

[25] E. Witten, "Quantum field theory and the Jones polynomial," Commun. Math. Phys. 121, 351 (1989).

[26] R. Gopakumar and C. Vafa, "Topological gravity as large $N$ topological gauge theory," Adv. Theor. Math. Phys. 2, 413 (1998) arXiv:hep-th/9802016.

[27] H. Ooguri and C. Vafa, "Knot invariants and topological strings," Nucl. Phys. B 577, 419 (2000) arXiv:hep-th/9912123.

[28] K. Liu and P. Peng, "Proof of the Labastida-Marino-Ooguri-Vafa conjecture," arXiv:0704.1526 [math.QA].

[29] M. Aganagic, A. Klemm, M. Marino and C. Vafa, "The topological vertex," Commun. Math. Phys. 254, 425 (2005) [arXiv:hep-th/0305132.

[30] M. Marino, "Chern-Simons theory, matrix models, and topological strings," ( Oxford, UK: Clarendon ,2005).

[31] H. Ooguri and C. Vafa, "Worldsheet derivation of a large $N$ duality," Nucl. Phys. B 641, 3 (2002) arXiv:hep-th/0205297.

[32] J. M. Maldacena, "The large $N$ limit of superconformal field theories and supergravity," Adv. Theor. Math. Phys. 2, 231 (1998) [Int. J. Theor. Phys. 38, 1113 (1999)] arXiv:hep-th/9711200.

[33] O. Aharony, S. S. Gubser, J. M. Maldacena, H. Ooguri and Y. Oz, "Large $N$ field theories, string theory and gravity," Phys. Rept. 323, 183 (2000) arXiv:hep-th/9905111.

[34] E. Witten, "Topological Sigma Models," Commun. Math. Phys. 118, 411 (1988).

[35] J. Distler and B. R. Greene, "Some exact results on the superpotential from CalabiYau compactifications," Nucl. Phys. B 309, 295 (1988). 
[36] S. W. Hawking, "Gravitational radiation from colliding black holes," Phys. Rev. Lett. 26, 1344 (1971)

[37] J. D. Bekenstein, "Black holes and entropy," Phys. Rev. D 7, 2333 (1973).

[38] S. W. Hawking, "Black hole explosions," Nature 248, 30 (1974).

[39] J. Polchinski, "Dirichlet-branes and Ramond-Ramond charges," Phys. Rev. Lett. 75, 4724 (1995) arXiv:hep-th/9510017.

[40] A. Strominger and C. Vafa, "Microscopic Origin of the Bekenstein-Hawking Entropy," Phys. Lett. B 379, 99 (1996) arXiv:hep-th/9601029].

[41] G. Lopes Cardoso, B. de Wit and T. Mohaupt, "Corrections to macroscopic supersymmetric black-hole entropy," Phys. Lett. B 451, 309 (1999) arXiv:hep-th/9812082.

[42] H. Ooguri, A. Strominger and C. Vafa, "Black hole attractors and the topological string," Phys. Rev. D 70, 106007 (2004) arXiv:hep-th/0405146.

[43] K. Becker, M. Becker and A. Strominger, "Five-branes, membranes and nonperturbative string theory," Nucl. Phys. B 456, 130 (1995) arXiv:hep-th/9507158.

[44] H. Ooguri, Y. Oz and Z. Yin, "D-branes on Calabi-Yau spaces and their mirrors," Nucl. Phys. B 477, 407 (1996) arXiv:hep-th/9606112.

[45] C. Vafa and E. Witten, "A Strong coupling test of S duality," Nucl. Phys. B 431, 3 (1994) arXiv:hep-th/9408074.

[46] H. Nakajima, "Instantons on ALE spaces, quiver varieties, and Kac-Moody algebras," Duke Math. 76, 365 (1994).

[47] C. Vafa, "Two dimensional Yang-Mills, black holes and topological strings," arXiv:hepth/0406058.

[48] M. Aganagic, H. Ooguri, N. Saulina and C. Vafa, "Black holes, $q$-deformed 2d YangMills, and non-perturbative topological strings," Nucl. Phys. B 715, 304 (2005) arXiv:hep-th/0411280.

[49] A. Dabholkar, F. Denef, G. W. Moore and B. Pioline, "Precision counting of small black holes," JHEP 0510, 096 (2005) arXiv:hep-th/0507014.

[50] D. Gaiotto, A. Strominger and X. Yin, "From $\mathrm{AdS}_{3} / \mathrm{CFT}_{2}$ to black holes / topological strings," JHEP 0709, 050 (2007) arXiv:hep-th/0602046.

[51] R. Dijkgraaf, C. Vafa and E. Verlinde, "M-theory and a topological string duality," arXiv:hep-th/0602087.

[52] C. Beasley, D. Gaiotto, M. Guica, L. Huang, A. Strominger and X. Yin, "Why $Z_{\mathrm{BH}}=$ $\left|Z_{\text {top }}\right|^{2}, "$ arXiv:hep-th/0608021.

[53] J. de Boer, M. C. N. Cheng, R. Dijkgraaf, J. Manschot and E. Verlinde, "A farey tail for attractor black holes," JHEP 0611, 024 (2006) [arXiv:hep-th/0608059].

[54] F. Denef and G. W. Moore, "Split states, entropy enigmas, holes and halos," arXiv:hep-th/0702146.

[55] R. K. Gupta and A. Sen, "AdS $3 / \mathrm{CFT}_{2}$ to $\mathrm{AdS}_{2} / \mathrm{CFT}_{1}$," arXiv:0806.0053 [hep-th]. 
[56] A. Okounkov, N. Reshetikhin and C. Vafa, "Quantum Calabi-Yau and classical crystals," arXiv:hep-th/0309208.

[57] S. K. Donaldson and R. P. Thomas, "Gauge theory in higher dimensions," in the Geometric Universe (Oxford Univ. Press, 1998).

[58] A. Iqbal, N. Nekrasov, A. Okounkov and C. Vafa, "Quantum foam and topological strings," JHEP 0804, 011 (2008) arXiv:hep-th/0312022.

[59] D. Maulik, A. Oblomkov, A. Okounkov, and R. Pandharipande, "Gromov-Witten/DonaldsonThomas correspondence for toric 3-folds," arXiv:math.AG/0809.3976.

[60] N. Nekrasov and A. Okounkov, "Seiberg-Witten theory and random partitions," arXiv:hep-th/0306238.

[61] R. Gopakumar and C. Vafa, "M-theory and topological strings. I," arXiv:hepth/9809187.

[62] R. Gopakumar and C. Vafa, "M-theory and topological strings. II," arXiv:hepth/9812127.

[63] B. Szendröi, "Non-commutative Donaldson-Thomas theory and the conifold," Geom. Topol. 12, 1171 (2008) [arXiv:0705.3419 [math.AG]].

[64] S. Mozgovoy and M. Reineke, "On the noncommutative Donaldson-Thomas invariants arising from brane tilings," arXiv:0809.0117 [math.AG].

[65] H. Ooguri and M. Yamazaki, "Crystal Melting and Toric Calabi-Yau Manifolds," arXiv:0811.2801 [hep-th].

[66] W. Heisenberg and W. Pauli, "Zur Quantendynamik der Wellenfelder," Z. Phys. 56, 1 (1929). 